\title{
Identifying, rating, and remembering environmental sound events
}

\author{
Michael Marcell, Maria Malatanos, Connie leahy, and Cadie Comeaux \\ College of Charleston, Charleston, South Carolina
}

\begin{abstract}
Sound events are sequences of closely grouped and temporally related environmental sounds that tell a story or establish a sense of place. The goal of our project was to create a set of sound events depicting various scenarios (such as a car accident, cooking breakfast, and walking outdoors) and to gather normative data about how people understand them. Samples of college students listened to 22 sound events over headphones in three self-paced, computer-based studies. In the Identification Task, 43 participants used text boxes to type descriptions of what was happening in the sound events. In the Rating Task, 39 participants used Likert scales to rate the sound events on the attributes of familiarity, complexity, and pleasantness. In the Memory Task, 42 participants answered two multiple-choice questions immediately after listening to each sound event. Detailed tables are provided for the following: (1) Description of the sound events and their components; (2) accuracy and response time measurements for each of the 22 sound events across the three studies; and (3) rank-orderings of the sound events by ease of identification, recognition of details, and rated familiarity, complexity, and pleasantness. Digital files of the stimuli, which may be of interest to auditory cognition researchers and clinical neuropsychologists, may be downloaded from either www.psychonomic.org/archive or www.cofc.edu/ marcellm/sound event studies/sndevent.htm.
\end{abstract}

Environmental sounds are nonmusical, nonlinguistic sounds that, according to Van Derveer (1979), Ballas and Howard (1987), and Jenkins (1985), have the following properties: (1) They are produced by real events (e.g., they are not machine-generated pure tones) and derive their meaning from those events; (2) they are typically more complex than sounds produced in the laboratory; (3) they can be animate (e.g., sigh) or inanimate (e.g., camera), natural (e.g., wind) or artificial (e.g., car horn) and (4) they are dynamic and convey, in Jenkins's words, "news that something is happening" (p. 117). Research on environmental sound identification and recognition has occurred primarily in the areas of cognitive psychology (e.g., Ballas, 1993; Fabiani, Kazmerski, Cycowicz, \& Friedman,1996; Gaver, 1993; Marcell, Borella, Greene, Kerr, \& Rogers, 2000) and clinical neuropsychology (e.g., Eustache, Lechevalier, Viader, \& Lambert, 1990; Schnider, Benson, Alexander, \& Schnider-Klaus, 1994; Van Lancker et al. 1988; Varney, 1980). Normed sets of simple environmental sounds (e.g., thunder, sneeze, phone ringing, rooster crowing, water draining) are available for research and clinical uses.

Previous research has focused on the identification of relatively brief, isolated environmental sounds; in contrast, less is known about how people perceive and understand complex, coherent, and lengthy sequences of natural sounds, such as the sounds accompanying the feeding of a cat or the fixing of a leaky faucet. Following McAdams and Bigand (1993), we refer to these lengthier sequences of closely grouped and temporally related sounds as "sound events." Their meaning unfolds over time as their nested component sounds contribute information and structure to the emergent event (cf. Ballas \& Howard, 1987; Gaver, 1993; McAdams, 1993; Warren \& Shaw, 1985). In the present study, we report the outcome of a project to determine how individuals identify, rate, and remember 22 sound events. Paralleling an earlier effort with 120 simpler environmental sounds (Marcell et al., 2000), our goal was to create an online archive of normed sound events for use in psychological research on complex auditory cognition and clinical neuropsychology research on auditory agnosia, word-finding difficulty, and elicited speech.

\section{METHOD}

\section{Participants}

One hundred twenty-four undergraduate psychology students at the College of Charleston, a southeastern liberal arts college, participated for either extra credit or fulfillment of a class requirement. $67.7 \%$ of the participants were female and $32.3 \%$ male, which is similar to the ratio at the College. Their mean age was 19.5 years $(S D=3.0)$.

As part of the computer-based testing protocol, each participant completed three self-report measures and five demographic and background questions (the sex and age items are summarized above). These measures are briefly described below in order to provide a more complete description of the self-reported characteristics of the participants at the time of their testing.

M. Marcell, marcellm@cofc.edu 
Hearing Screening Inventory (HSI; Coren \& Hakstian, 1992). The HSI is a self-report auditory screening measure that is used to assess the degree of hearing loss. The HSI is composed of 12 questions that probe how well one hears in everyday situations. HSI scores range from 12-60, with scores between 12-27 classified as normal, 28-37 as mild hearing loss, and 38-60 as moderate-tosevere loss. The mean HSI score of the participants in this study was $21.9(S D=5.1)$, which is well within the normal hearing range.

Auditory Imagery Scale (AIS; Gissurarson, 1992). The AIS is a self-report measure that is used to assess how well participants form images of sounds. The AIS consists of seven questions regard-

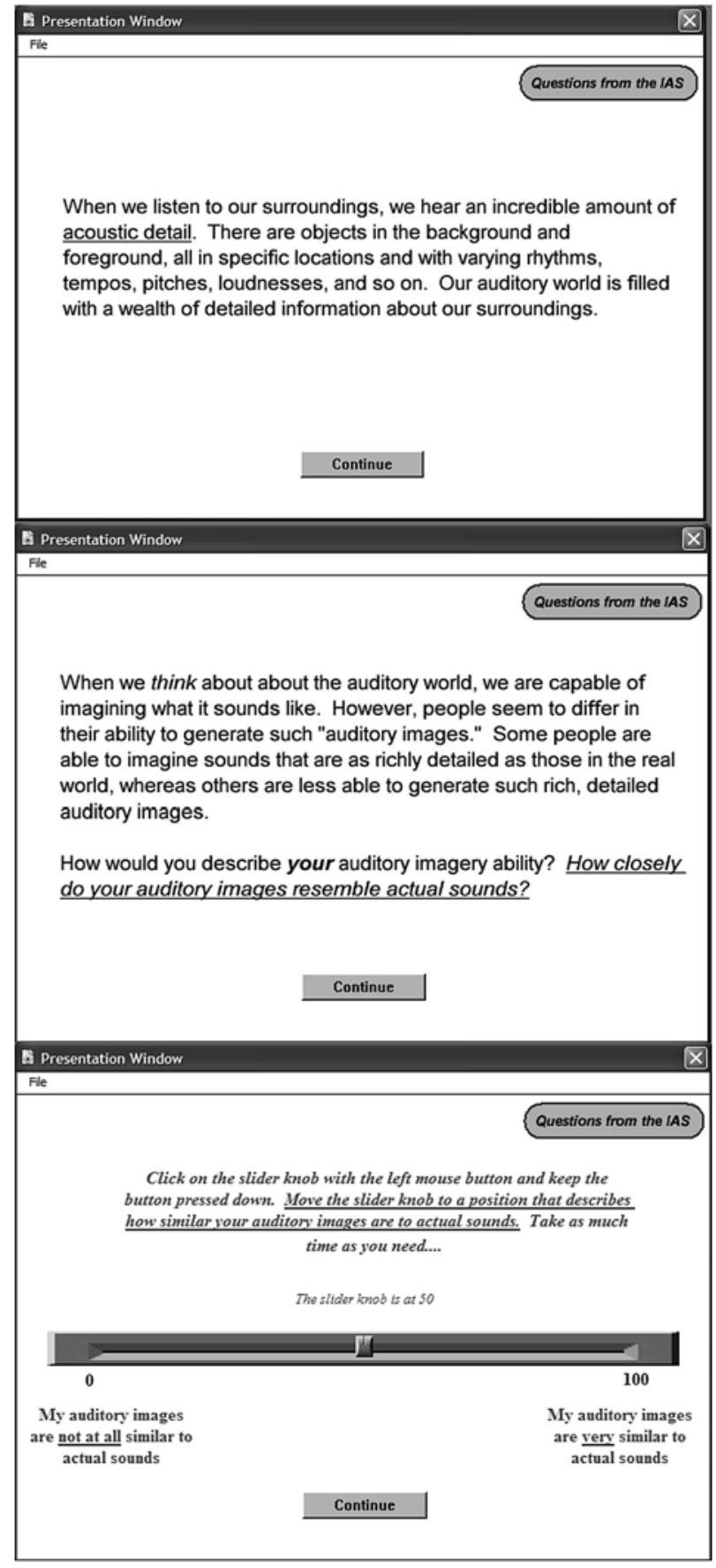

Figure 1. Screenshots of Imagery Analogue Scale (auditory). ing the vividness of auditory imagery. AIS scores range from 7 to 28 , with lower scores indicating clearer auditory imagery. The mean AIS score of the participants in this study was $11.0(S D=3.1)$. In Gissurason's study, 160 participants had a mean score of 12.0 ( $S D=$ 3.6 ), suggesting that ratings of strong auditory imagery may be the norm for this self-report inventory.

Imagery Analogue Scales (IAS; Marcell, 2006). The IAS was developed to provide rapid self-report estimates of auditory and visual imagery ability. Screenshots of the auditory and visual components of the IAS can be found in Figures 1 and 2, respectively. Briefly, participants were given working definitions of "auditory imagery" and "visual imagery" that stressed the ability to imagine sensory details vividly and accurately. Afterward, they reported on their ability to create detailed images by positioning a knob on a slider scale ranging from $0-100$, with higher numbers indicating that images are very similar to actual sounds or sights. The mean auditory IAS score of the participants was 75.6 $(S D=15.8)$ and the mean visual IAS score was $79.7(S D=15.5)$. Participants tended to report that they have vivid auditory and visual imagery, with their estimates of visual imagery slightly higher $[t(123)=2.593, p=$ $.011,2$-tailed, $d=0.23$ (small effect size)]

Background questions. Participants responded to the question, "How would you rate your own musical ability, regardless of your formal musical training?" on a 7-point Likert scale, with 1 indicating very poor, 4 average, and 7 very good. The mean rating was $4.2(S D=1.7)$, suggesting that the typical participant considered herself or himself of average musical ability. Participants were also asked to estimate the amount of their musical training by responding to the multiple-choice question, "How many years of formal musical training (such as years in school band, piano lessons, or voice lessons) have you had?" Responses were distributed evenly among the four categories, with $29.0 \%$ indicating that they had 0 years of musical training, $21.0 \% 1-2$ years, $23.4 \% 3-4$ years, and $26.6 \% 5$ or more years. Finally, participants were asked to rate the number of distractions in their current surroundings on a 7-point Likert scale, with 1 indicating a very low rating, 4 average, and 7 very high. The mean rating was $2.4(S D=1.2)$, suggesting that participants did not find themselves distracted during the course of the testing session.

\section{Apparatus, Materials, and Procedure}

Apparatus and Stimuli. Participants were tested in a psychology laboratory classroom on Pentium-based computers equipped with 17inch monitors and headphones. The stimuli were 2 practice and 22 experimental "sound events," which were defined as coherent sequences of environmental sounds that tell a simple story or establish a sense of place. An example of a sound event that tells a simple story is the "baby photos" stimulus, in which the overlapping sounds of a crying baby and a photographer's camera were followed in sequence by the sound of jingling keys, the cooing sounds of a happy baby, and more camera sounds. The sequence suggests that the keys were jingled in order to improve the mood of the baby during a photography session. An example of a sound event that establishes a sense of place is the "bathroom chores" stimulus, in which the related activities of brushing teeth, running water, gargling, and a toilet flushing suggests an individual in a bathroom who is engaged in routine personal hygiene.

The constituent environmental sounds came from several sources: Sound effects collections on CD-ROM (Innovative Media Corp., 1993; Wayzata Technology, 1995), Marcell et al.'s (2000) online archive of 120 environmental sounds, and WWW sound archives that offer free digital samples for downloading (e.g., www.alfreesoundeffects.com). Like Marcell et al. (2000), we attempted to select environmental sounds that were clear, realistic, and potentially identifiable. Individual sounds were blended into lengthier sound events via Goldwave sound-editing software. Examples of the types of edits performed included cutting silences, pasting repetitions, changing volume and pitch, sequencing overlapping sounds on separate channels, and fading out sounds. An attempt was made to equate the volume levels of the stimuli by setting all sound events to the same RMS (root mean square) level and then subjectively 
equating the RMS-adjusted sounds. Sound sequences were edited in stereo format and saved as 16-bit mono.WAV files.

Table 1 provides a detailed listing of the sequence of component sounds in each of the 24 sound events. The number of component sounds in the experimental stimuli ranged from 3 to 10 , with an average of 5.6 component sounds per sound event $(S D=1.9) .{ }^{1}$ Also, as Table 1 shows, experimental sound events ranged in duration from 8.7 to $36.5 \mathrm{sec}$, with an average length of $18.3 \mathrm{sec}(S D=7.4)$.

General testing procedure. Stimuli were presented via three computer programs created with Macromedia Authorware, and each participant was randomly assigned to complete one of the programs during a

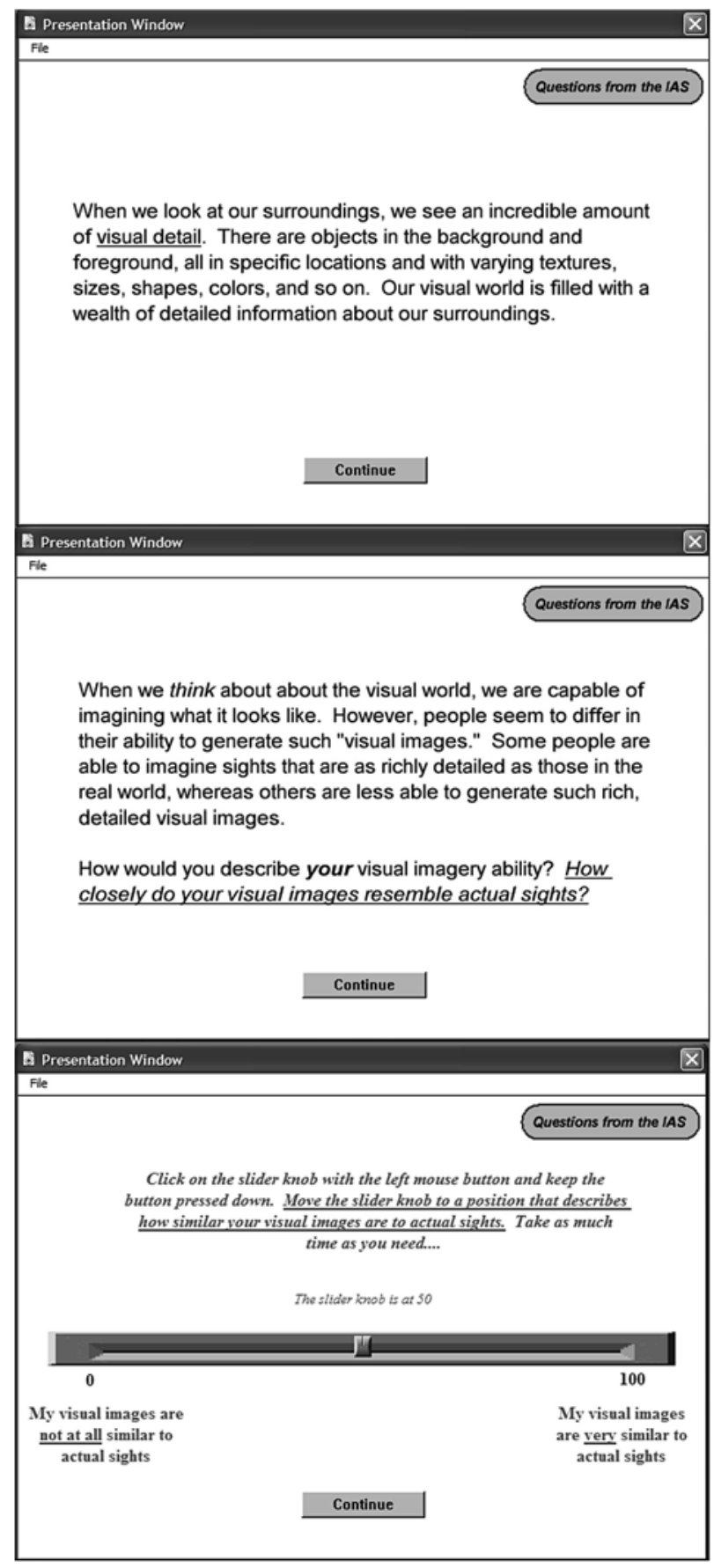

Figure 2. Screenshots of Imagery Analogue Scale (visual). single testing session. All tasks administered during the testing session were self-paced, instructions presented on easy-to-read screens, and sounds presented over headphones. Participants were tested in small groups ranging in size from 1-6 individuals. A testing session began with the experimenter greeting the participant(s), obtaining informed consent, and providing a brief orientation to the equipment used in the study. Afterward, the following steps occurred in each computer program: (1) Participants entered a name of their choice (e.g., a nickname) into a text box. The program appended a random number to the name to create a unique user ID that accompanied the entry of data into the server database. (2) A screen appeared for 5-10 sec stating that information about the participant's computer was being recorded (such as the type of operating system, browser version, and results of timing accuracy checks). (3) The three self-report measures (HSI, AIS, and IAS) were presented in a random order. (4) Instructions appeared that asked participants to don their headphones and adjust the loudness of the playback to a comfortable level while listening to a continuous loop of environmental sounds. (5) Instructions and two practice trials were presented for one of the three randomly selected sound event tasks (identification, rating, or memory). (6) The 22 experimental stimuli were presented in a random order. Each sound event was played twice with a 1-sec interval between the repetitions, and participants had an unlimited amount of time to respond. (7) After the task was completed, a screen appeared informing participants that they could remove their headphones. Randomly ordered questions about sex, age, musical ability, musical training, and number of distractions in the current environment were then presented. (8) The computer timing checks from Step (2) were repeated. (9) A textbox was provided for the participant to provide feedback about the study. (10) The participant's raw data were displayed for inspection. (11) A debriefing screen was presented in which participants were thanked and given an overview of the purpose and goals of the study.

The testing session concluded with a personal thank-you from the experimenter and an opportunity for the participant to ask the experimenter questions about the project.

Sound event tasks. The 43 participants in the identification task were asked to "'tell the story' of the sound event - to describe what is happening in the sequence of sounds." They were asked to incorporate as many of the individual component sounds as they could into their typed description of a sound event, and they were reminded to be "accurate and complete" in their description. At the beginning of the second playing of a sound event, participants were presented a text box in which they were asked to type a description of the sound event. They had an unlimited amount of time to type their description and could begin doing so anytime during or after the second playing of the sound event. Immediately after each practice trial was completed, an experimentergenerated "prototypical" description of the sound event was displayed alongside the participant's identification response, and the participant was encouraged to compare the accuracy and detail of the two descriptions. No such feedback was provided during the experimental trials.

Data were gathered for each sound event on the content of the typed identification response and the typing response time. The content of an identification response was independently evaluated by two experimenters who awarded points for the following: (1) Reporting the key sound components; (2) listing certain components in their correct order, and/or (3) making correct inferences about the sound event (e.g., recognizing that the jingling sound made the baby happy). Guidelines for awarding points were developed using the procedures of Marcell et al. (2000) (e.g., spelling and grammatical errors, such as the use of word fragments and incomplete sentences, were ignored). Disagreements between the two experimenters were used to refine and reword the scoring criteria. Criteria used to score identification responses for each sound event can be found in an archived .pdf table posted at www.psychonomic.org/archive.

Two response time variables were measured by the computer: (1) "First letter RT" was the time between the onset of the text box (which occurred simultaneously with the end of the first playing of the sound event) and the typing of the first letter of the identification 
response; (2) "Full RT" was the time between the onset of the text box to the completion of the typed response, which was signaled by pressing the Enter key. Participants required an average of $29.6 \mathrm{~min}$ $(S D=7.0)$ to complete the identification task and an additional $6.5 \mathrm{~min}$ to complete the other components of their testing session.

The 39 participants in the rating task were given directions to rate each sound on the attributes of familiarity, complexity, and pleasantness. At the beginning of the second playing of a sound event, a screen appeared containing both a brief description of one of the three attributes and a 7-point Likert scale (see Figure 3). After rating the sound event on the attribute, similar screens appeared for rating the other two attributes. The three rating scales were presented in a random order across participants but remained in the same order for a given participant throughout the task. Participants had an unlimited amount of time to rate a sound event and could begin doing so anytime during or after the second playing of the stimulus. Although feedback in the form of simple reminders of the numerical ratings they had assigned was provided after each practice trial, no feedback was presented on the experimental trials. The dependent variables gathered during the rating task were familiarity, complexity, and pleasantness ratings and the total time needed to complete the three ratings for a sound event (measured from the onset of the first rating scale to the completion of the third rating scale). Participants required an average of $17.1 \mathrm{~min}$ $(S D=2.8)$ to complete the rating task and an additional $7.5 \mathrm{~min}$ to complete the other components of their testing session.

The 42 participants in the memory task were instructed to "listen very carefully and try to remember what is happening in the sequence of sounds" so that they could later answer two questions about it. Immediately after the second playing of a sound event, participants were presented two, four-alternative multiple-choice questions about some aspect of the event and asked to answer them as best as they could (e.g., one of the questions for the "car crash" sound event was as follows: "Where did this accident most likely take place? (1) parking lot; (2) highway (the correct answer); (3) country road; (4) city street"). The questions were presented sequentially and in a random order. The position of the correct answer (whether it was the first-listed alternative, second-listed alternative, and so on) was counterbalanced so that each of the four positions was used as the correct alternative 12 times across the set of 48 questions. Feedback was provided on the number of correct answers during both the practice and experimental trials. The set of multiple-choice questions used in the memory task can be found in an archived .pdf table posted at www.psychonomic .org/archive.

The dependent variables collected in the memory task were the accuracy of a participant's answer to each question, the number of questions correctly answered per sound event, and the total time needed to answer both questions. The accuracy data were later converted to memory scores representing the percentage of the sample correctly answering a given question. Participants required an average of $25.1 \mathrm{~min}$ $(S D=1.9)$ to complete the memory task and an additional $6.0 \mathrm{~min}$ to complete the other components of their testing session.

The computer programs for the identification task, rating task, and memory task are posted online at www.cofc.edu/ marcellm/ sound event studies/sndevent.htm. Running the software programs

Table 1

Durations and Component Sounds of the Sound Events

\begin{tabular}{|c|c|c|}
\hline Sound Event & Duration $(\mathrm{sec})$ & Component Sounds (in Order of Occurrence) \\
\hline & & Practice Stimuli \\
\hline Bedtime reading & 11.55 & Pages turning, cuckoo clock chiming three times, thumping noise, clicking of lamp switch \\
\hline \multirow[t]{2}{*}{ Going boating } & 18.68 & Boat engine idling, seagulls, waves hitting boat, boat horn (twice) \\
\hline & & Experimental Stimuli \\
\hline Assembling papers & 12.24 & $\begin{array}{l}\text { Turning pages (twice), cutting paper with scissors (twice), stapling of papers, tearing paper, } \\
\text { crumpling paper }\end{array}$ \\
\hline Baby photos & 8.69 & Baby crying, camera sounds, keys jingling, baby cooing, camera sounds \\
\hline Bathroom chores & 23.99 & Brushing teeth, running water, gargling (twice), shower running, toilet flushing \\
\hline Car chase & 28.67 & $\begin{array}{l}\text { Person running, car door slamming, car starting, shifting into gear, car leaving quickly, traffic } \\
\text { sounds, person running, car door slamming, car starting, car leaving quickly }\end{array}$ \\
\hline Car crash & 10.28 & Traffic noises, horn blowing several times, skidding, two cars crashing \\
\hline Casino sounds & 24.59 & $\begin{array}{l}\text { Casino ambience, shuffling of cards, slot machine sounds, roulette wheel sounds, coin dropping, } \\
\text { talking in background throughout }\end{array}$ \\
\hline City sounds & 24.24 & $\begin{array}{l}\text { Sawing, drilling, jackhammer sounds throughout, people walking on sidewalk, people talking, } \\
\text { bus sounds, horns blowing in traffic jam }\end{array}$ \\
\hline Coming home & 12.66 & $\begin{array}{l}\text { Keys jingling, unlocking door, squeaky door opening, door closing, person's footsteps, phone } \\
\text { ringing }\end{array}$ \\
\hline Cooking breakfast & 36.46 & $\begin{array}{l}\text { Rooster, pouring liquid, swallowing, water running, moving objects in refrigerator, closing re- } \\
\text { frigerator, handling metal pots, frying food, tea kettle whistling, handling dishes and pots }\end{array}$ \\
\hline Dinner party & 14.61 & $\begin{array}{l}\text { Group of people talking and laughing, cork popping, serving of dishes, tapping on glass, clap- } \\
\text { ping, doorbell ringing, people talking, door closing }\end{array}$ \\
\hline Dog damage & 10.99 & Dog barking, glass breaking, person running, dog whimpering, woman crying \\
\hline Drinking soda & 12.39 & Opening "pop-top" can, guzzling and gulping sounds, crushing can, burping \\
\hline Dripping faucet & 19.08 & $\begin{array}{l}\text { Yawning, clicking of lamp switch, dripping, clicking of lamp switch, footsteps, tightening } \\
\text { squeaky faucet }\end{array}$ \\
\hline Feeding cat & 17.84 & $\begin{array}{l}\text { Cat meowing, opening can with manual can opener, placing can opener on counter, putting food } \\
\text { on plate, scraping out can }\end{array}$ \\
\hline Nighttime camping & 19.93 & Frogs croaking and crickets chirping throughout, zipping tent, yawning, mosquito buzzing \\
\hline Ocean vessel & 27.62 & $\begin{array}{l}\text { Seagulls squawking throughout, ocean waves throughout, fog horn getting louder over three repeti- } \\
\text { tions as vessel approaches }\end{array}$ \\
\hline Ping-Pong accident & 11.10 & Playing Ping-Pong, glass breaking, running, door slamming \\
\hline Playing basketball & 12.17 & $\begin{array}{l}\text { Four dribbles, basketball hitting backboard, four dribbles, basketball hitting backboard and going } \\
\text { through net }\end{array}$ \\
\hline Sick man & 12.48 & Loud sneeze, sniffling, blowing nose, coughing, water running, gulping \\
\hline Thunderstorm approaching & 20.49 & Strong wind, wind chimes, closing two windows, thunder in the distance \\
\hline Typing interruptions & 17.88 & Typing, baby crying, woman sighing, typing, doorbell ringing, baby fussing, woman sighing \\
\hline Walking outdoors & 24.96 & Forest sounds (e.g., birds, insects), walking on leaves, walking on gravel, gunshot in the distance \\
\hline
\end{tabular}




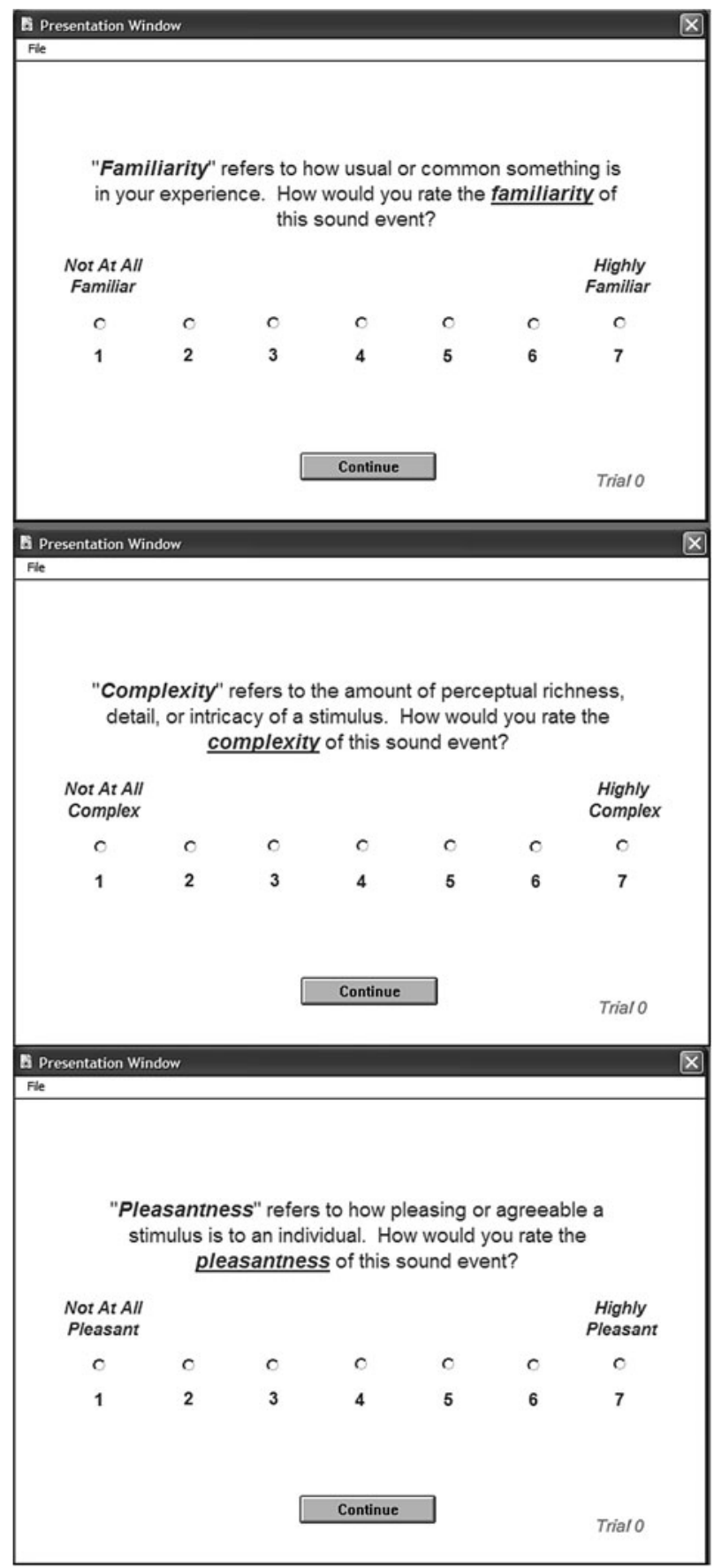

Figure 3. Screenshots of Sound Events Ratings scales.

over the Web requires the installation of a free Macromedia Authorware browser plugin.

\section{RESULTS AND DISCUSSION}

For the inferential statistics reported in this section, all significance tests were two-tailed, alpha set at .05, and effect sizes interpreted using Cohen's (1988) guidelines. Table 2 presents summary descriptive statistics for each of the 22 experimental sound events. On the identification task, participants earned an average of two-thirds $(M=$ $66.9 \%, S D=13.4)$ of the available points when identifying a sound event, required an average of $3.1 \mathrm{sec}(S D=1.0)$ to begin typing their identification response, and completed a typical response in $44.3 \mathrm{sec}(S D=7.1)$. Correlational analyses revealed that more accurate identification responses were initiated more quickly $[r(20)=-.508, p<.05]$, a pattern that was reported by Marcell et al. (2000) for vocal response times to brief environmental sounds. Also, identification responses took longer to complete when the sound events were lengthier $[r(20)=.697, p<.01]$ or contained a larger number of key components $[r(20)=.406, p=.06]$ (marginally significant). As Marcell et al. noted, this is to be expected given that a longer event requires more time, and thus more listening, for the sound event to unfold (and for the individual to describe it).

Table 3 presents a ranking of sound events according to the completeness of the identification responses. Participants gave the most complete identification responses to the "baby photos," "bathroom chores," "coming home," and "drinking soda" sound events and the least complete responses to the "walking outdoors," "casino sounds," "dripping faucet," and "city sounds" sound events. It is important to remember that conclusions concerning the completeness of participants' identification responses are tied in part to the particular scoring guidelines, which were influenced by our expectations of what constitutes a good response. It is possible that other scoring systems (e.g., a system that counts only keywords, or a system that requires the scorer to assign a Likert-style global rating of the quality of a response) would yield different outcomes that might be more appropriate for specific applications. Like Marcell et al. (2000), we encourage users of our sound event stimuli to continue to refine the proposed identification scoring guidelines and to explore the development of alternative scoring systems. ${ }^{2}$

Analyses of the rating task (see Table 2) indicated that the sound events were generally considered to be familiar $(M=5.3, S D=0.7)$, average in complexity $(M=4.4$, $S D=0.7)$, and slightly unpleasant $(M=3.3, S D=1.1)$. Participants completed the three rating scales quickly $(M=$ $14.2 \mathrm{sec}, S D=1.4 \mathrm{sec}$ ), and completed them faster when the sound events were more familiar $[r(20)=-.537, p<$ $.01]$ or had fewer key components $[r(20)=.403, p=.06]$ (marginally significant). Also, participants tended to rate longer sound events as more complex $[r(20)=.599, p<$ $.01]$ and more pleasant $[r(20)=.432, p<.05]$, a finding which was also reported for Marcell et al.'s (2000) set of 120 environmental sounds. Finally, sound events with fewer key components tended to be rated as more familiar $[r(20)=-.411, p=.058]$ (marginally significant). The absence of significant correlations among the attributes of familiarity, complexity, and pleasantness suggests that raters treated these dimensions independently.

The high familiarity ratings for the set of stimuli paralleled those reported by Marcell et al. (2000) for their set of simple environmental sounds and Snodgrass and Vanderwart (1980) for their set of line drawings. As can be seen in Table 4, the four most familiar sound events were "bathroom chores," "playing basketball," "thunder- 


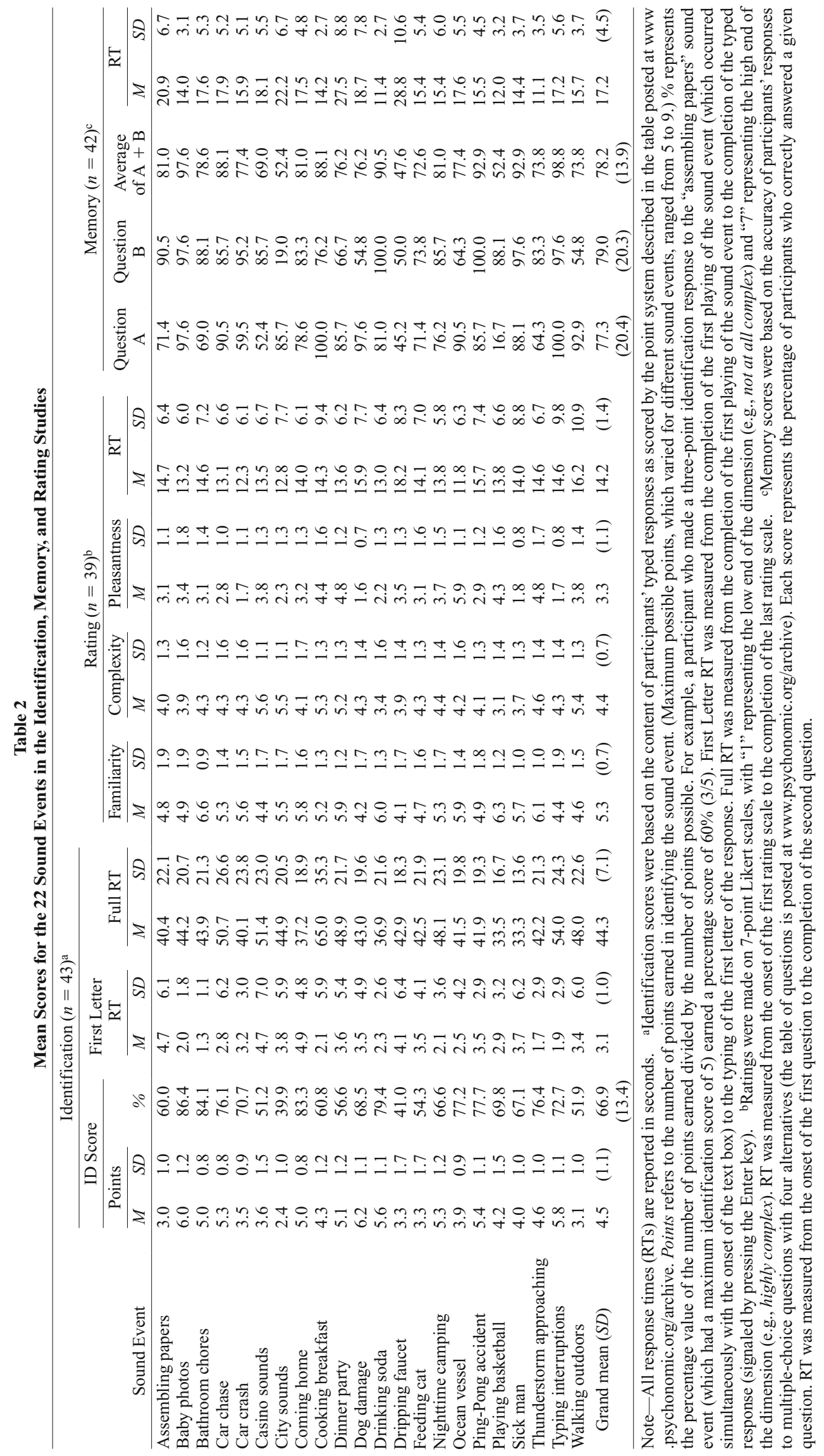


Table 3

Sound Events Listed in Descending Order of Completeness of Identification Responses

\begin{tabular}{|c|c|}
\hline Sound Event & $\begin{array}{l}\text { Completeness } \\
\text { of Identification } \\
\text { Response }(\%)\end{array}$ \\
\hline Baby photos & 86.4 \\
\hline Bathroom chores & 84.1 \\
\hline Coming home & 83.3 \\
\hline Drinking soda & 79.4 \\
\hline Ping-Pong accident & 77.7 \\
\hline Ocean vessel & 77.2 \\
\hline Thunderstorm approaching & 76.4 \\
\hline Car chase & 76.1 \\
\hline Typing interruptions & 72.7 \\
\hline Car crash & 70.7 \\
\hline Playing basketball & 69.8 \\
\hline Dog damage & 68.5 \\
\hline Sick man & 67.1 \\
\hline Nighttime camping & 66.6 \\
\hline Cooking breakfast & 60.8 \\
\hline Assembling papers & 60.0 \\
\hline Dinner party & 56.6 \\
\hline Feeding cat & 54.3 \\
\hline Walking outdoors & 51.9 \\
\hline Casino sounds & 51.2 \\
\hline Dripping faucet & 41.0 \\
\hline City sounds & 39.9 \\
\hline
\end{tabular}

Note-It should be remembered that the completeness of a response is based on the amount of information recalled from the sound event relative to the amount of information that we anticipated should be recalled.

storm approaching," and "drinking soda," and the four least familiar sound events were "casino sounds," "typing interruptions," "dog damage," and "dripping faucet" (it seems reasonable to suspect that the sounds of casinos, typewriters, and old-fashioned plumbing might be relatively unfamiliar to contemporary college students). Table 5 reveals that the most complex sound events were "casino sounds," "city sounds," "walking outdoors," and

Table 4

Sound Events Listed in Descending Order of Familiarity

\begin{tabular}{lc}
\hline \multicolumn{1}{c}{ Sound Event } & Mean Rating \\
\hline Bathroom chores & 6.6 \\
Playing basketball & 6.3 \\
Thunderstorm approaching & 6.1 \\
Drinking soda & 6.0 \\
Dinner party & 5.9 \\
Ocean vessel & 5.9 \\
Coming home & 5.8 \\
Sick man & 5.7 \\
Car crash & 5.6 \\
City sounds & 5.5 \\
Car chase & 5.3 \\
Nighttime camping & 5.3 \\
Cooking breakfast & 5.2 \\
Baby photos & 4.9 \\
Ping-Pong accident & 4.9 \\
Assembling papers & 4.8 \\
Feeding cat & 4.7 \\
Walking outdoors & 4.6 \\
Casino sounds & 4.4 \\
Typing interruptions & 4.4 \\
Dog damage & 4.2 \\
Dripping faucet & 4.1 \\
\hline
\end{tabular}

Note-Stimuli with tied familiarity ranks are listed alphabetically. "cooking breakfast," and the simplest sound events were "baby photos" and "dripping faucet" (tied), "sick man," "drinking soda," and "playing basketball." Given the previously reported positive correlation between rated complexity and stimulus duration, it is not surprising that the complex sound events listed above were significantly longer $(M=27.6 \mathrm{sec}, S D=5.9 \mathrm{sec})$ than the simple sound events listed above $(M=13.0 \mathrm{sec}, S D=3.8 \mathrm{sec})[t(7)=$ $4.52, p=.003]$. Finally, Table 6 indicates that the most pleasant sound events were "ocean vessel," "dinner party," "thunderstorm approaching," and "cooking breakfast," and the most unpleasant sound events were "sick man," "car crash," "typing interruptions," and "dog damage." One surprise was the large number of unpleasant sound events in the set: 17 of the 22 stimuli had mean pleasantness ratings lower than 4 , which was the middle position on the pleasantness rating scale.

On the memory task (see Table 2), participants showed an overall accuracy rate of $78.2 \%(S D=13.9)$ in answering four-alternative questions about sound events, a value that was significantly higher than chance expectation of $25 \%[t(21)=17.99, p<.000001, d=3.84$ (large effect size)]. Participants completed the two memory questions quickly $(M=17.2 \mathrm{sec}, S D=4.5 \mathrm{sec})$, and finished them faster when their answers were correct $[r(20)=-.424$, $p<.05]$ or when there were fewer key components in the sound event $[r(20)=.443, p<.05]$.

Fourteen of the 44 questions were answered correctly by over $90 \%$ of the participants, and only four questions were answered at accuracy rates under $50 \%$. Table 7 reveals that participants were most accurate in answering questions about the "typing interruptions," "baby photos," "Ping-Pong accident," and "sick man" sound events, and least accurate in answering questions about the "casino sounds," "city sounds," "playing basketball," and "dripping faucet" sound events. For two of the latter stimuli, low memory scores were caused

Table 5

Sound Events Listed in Descending Order of Complexity

\begin{tabular}{lc}
\hline \multicolumn{1}{c}{ Sound Event } & Mean Rating \\
\hline Casino sounds & 5.6 \\
City sounds & 5.5 \\
Walking outdoors & 5.4 \\
Cooking breakfast & 5.3 \\
Dinner party & 5.2 \\
Thunderstorm approaching & 4.6 \\
Nighttime camping & 4.4 \\
Bathroom chores & 4.3 \\
Car chase & 4.3 \\
Car crash & 4.3 \\
Dog damage & 4.3 \\
Feeding cat & 4.3 \\
Typing interruptions & 4.3 \\
Ocean vessel & 4.2 \\
Coming home & 4.1 \\
Ping-Pong accident & 4.1 \\
Assembling papers & 4.0 \\
Baby photos & 3.9 \\
Dripping faucet & 3.9 \\
Sick man & 3.7 \\
Drinking soda & 3.4 \\
Playing basketball & 3.1 \\
\hline
\end{tabular}

Note-Stimuli with tied complexity ranks are listed alphabetically. 
Table 6

Sound Events Listed in Descending Order of Pleasantness

\begin{tabular}{lc}
\hline \multicolumn{1}{c}{ Sound Event } & Mean Rating \\
\hline Ocean vessel & 5.9 \\
Dinner party & 4.8 \\
Thunderstorm approaching & 4.8 \\
Cooking breakfast & 4.4 \\
Playing basketball & 4.3 \\
Casino sounds & 3.8 \\
Walking outdoors & 3.8 \\
Nighttime camping & 3.7 \\
Dripping faucet & 3.5 \\
Baby photos & 3.4 \\
Coming home & 3.2 \\
Assembling papers & 3.1 \\
Bathroom chores & 3.1 \\
Feeding cat & 3.1 \\
Ping-Pong accident & 2.9 \\
Car chase & 2.8 \\
City sounds & 2.3 \\
Drinking soda & 2.2 \\
Sick man & 1.8 \\
Car crash & 1.7 \\
Typing interruptions & 1.7 \\
Dog damage & 1.6 \\
\hline
\end{tabular}

Note-Stimuli with tied pleasantness ranks are listed alphabetically.

Table 7

Sounds Events Listed in Descending Order of Overall Memory Score

\begin{tabular}{lc}
\hline \multicolumn{1}{c}{ Sound Event } & $\begin{array}{c}\text { Percent Correctly } \\
\text { Answering Questions }{ }^{\mathrm{a}}\end{array}$ \\
\hline Typing interruptions & 98.8 \\
Baby photos & 97.6 \\
Ping-Pong accident & 92.9 \\
Sick man & 92.9 \\
Drinking soda & 90.5 \\
Car chase & 88.1 \\
Cooking breakfast & 88.1 \\
Assembling papers & 81.0 \\
Coming home & 81.0 \\
Nighttime camping & 81.0 \\
Bathroom chores & 78.6 \\
Car crash & 77.4 \\
Ocean vessel & 77.4 \\
Dinner party & 76.2 \\
Dog damage & 76.2 \\
Walking outdoors & 73.8 \\
Thunderstorm approaching & 73.8 \\
Feeding cat & 72.6 \\
Casino sounds & 69.0 \\
City sounds & 52.4 \\
Playing basketball & 52.4 \\
Dripping faucet & 47.6 \\
\hline
\end{tabular}

Note-Stimuli with tied scores are listed alphabetically. a This value represents the percentage of times the sample correctly answered Questions $\mathrm{A}$ and $\mathrm{B}$.

by difficulties in answering only one of the two questions. Although one might argue that difficult questions should be replaced, we believe that a larger set of difficult questions is actually warranted and would be helpful in distinguishing "careful listeners" from "casual listeners." Future researchers might wish to develop additional questions, and to determine whether the completeness with which participants de- scribe an ongoing sound event correlates with the accuracy of their memory for details of the event.

In conclusion, we have developed a set of 22 nonlinguistic, nonmusical auditory stimuli of varying lengths and themes, each of which tells a simple story and/or establishes a sense of place. Normative data are available on how carefully listeners describe the sound events, how familiar, complex, and pleasant the events seem to listeners, and how accurately listeners answer questions about them from memory. We hope that these new materials will be of interest to researchers in auditory cognition and clinical neuropsychology, who might find it beneficial to study topics such as confrontation naming, word finding difficulty, and elicited speech using novel auditory stimuli (cf. suggestions by Marcell et al., 2000). We encourage researchers to freely download the digital sound event files posted online and to use them to explore auditory perceptual and cognitive phenomena of interest. If the stimuli prove useful, we also invite researchers to extend the normative data collection effort by collecting data on additional populations such as children and older adults.

\section{AUTHOR NOTE}

An earlier version of this project was presented as a poster at the August 2005 meeting of the American Psychological Association in Washington, DC. We thank the undergraduate psychology students at the College of Charleston who participated in our study. Questions, comments, or suggestions about this article should be directed to M. Marcell, Department of Psychology, College of Charleston, Charleston, SC 29424 (e-mail: marcellm@cofc.edu).

\section{REFERENCES}

Ballas, J. A. (1993). Common factors in the identification of an assortment of brief everyday sounds. Journal of Experimental Psychology: Human Perception \& Performance, 19, 250-267.

Ballas, J. A., \& Howard, J. H. (1987). Interpreting the language of environmental sounds. Environment \& Behavior, 19, 91-114.

COHEN, J. (1988). Statistical power analysis for the behavioral sciences. Hillsdale, NJ: Erlbaum.

Coren, S., \& HaKstian, A. R. (1992). The development and crossvalidation of a self-report inventory to assess pure-tone threshold hearing sensitivity. Journal of Speech \& Hearing Research, 35, 921-928.

Eustache, F., Lechevalier, B., Viader, F., \& Lambert, J. (1990). Identification and discrimination disorders in auditory perception: A report on two cases. Neuropsychologia, 28, 257-270.

Fabiani, M., Kazmerski, V. A., Cycowicz, Y. M., \& Friedman, D. (1996). Naming norms for brief environmental sounds: Effects of age and dementia. Psychophysiology, 33, 462-475.

Gaver, W. W. (1993). What in the world do we hear? An ecological approach to auditory event perception. Ecological Psychology, 5, 1-29.

GISSURARSON, L. R. (1992). Reported auditory imagery and its relationship with visual imagery. Journal of Mental Imagery, 16, 117-122.

GoodGLASS, H. (1980). Disorders of naming following brain injury. American Scientist, 68, 647-655.

Innovative Media Corporation (1993). Sonic Waves 3000. Springfield, IL: IMC Sonic Waves Production.

Jenkins, J. J. (1985). Acoustic information for objects, places, and events. In W. H. Warren Jr. \& R. E. Shaw (Eds.), Persistence and change: Proceedings of the First International Conference on Event Perception (pp. 115-138). Hillsdale, NJ: Erlbaum.

MARCELL, M. M. (2006). Imagery analogue scales (IAS). Unpublished data.

Marcell, M. M., Borella, D., Greene, M., Kerr, E., \& Rogers, S. (2000). Confrontation naming of environmental sounds. Journal of Clinical \& Experimental Neuropsychology, 6, 830-864. 
McAdams, S. (1993). Recognition of sound sources and events. In S. McAdams \& E. Bigand (Eds.), Thinking in sound: The cognitive psychology of human audition (pp. 146-198). Oxford: Oxford University Press, Clarendon Press.

McAdAms, S., \& Bigand, E. (1993). Introduction to auditory cognition. In S. McAdams \& E. Bigand (Eds.), Thinking in sound: The cognitive psychology of human audition (pp. 1-9). Oxford: Oxford University Press, Clarendon Press.

Schnider, A., Benson, D. F., Alexander, D. N., \& Schnider-Klaus, A. (1994). Non-verbal environmental sound recognition after unilateral hemispheric stroke. Brain, 117, 281-287.

SnOdgrass, J. G., \& VAnderwart, M. (1980). A standardized set of 260 pictures: Norms for name agreement, image agreement, familiarity, and visual complexity. Journal of Experimental Psychology: Human Learning \& Memory, 6, 174-215.

VAN DerVeER, N. J. (1979). Ecological acoustics: Human perception of environmental sounds (University Microfilms International No. 8004002). Dissertation Abstracts International.

Van Lancker, D., Cornelius, C., Kreiman, J., Tonick, I., Tanguay, P., \& Schulman, M. L. (1988). Recognition of environmental sounds in autistic children. Journal of the American Academy of Child \& Adolescent Psychiatry, 27, 423-427.

VARNEY, N. R. (1980). Sound recognition in relation to aural language comprehension in aphasic patients. Journal of Neurology, Neurosurgery, \& Psychiatry, 43, 71-75.

WARREN, W. H., \& SHAw, R. E. (1985). Events and encounters as units of analysis for ecological psychology. In W. H. Warren Jr. \& R.E. Shaw (Eds.), Persistence and change: Proceedings of the First International Conference on Event Perception (pp. 1-27). Hillsdale, NJ: Erlbaum.

Wayzata Technology (1995). Sound Library 2000. Grand Rapids, MI: Wayzata Technology.

\section{NOTES}

1. What constituted a "component sound" was often an arbitrary decision (e.g., should the boat horn sound in the "ocean vessel" sound event be counted as one or three components given that it occurred three separate times within the event? Should the background nature sounds in the "nighttime camping" sound event be subdivided into two component sounds, frogs croaking and crickets chirping?). Our decisions as to what constituted component sounds were practical and artistic rather than empirical in nature (cf. Marcell et al., 2000).

2. If sound event stimuli were used to evaluate the quality of an individual's expressive language, then one should develop a scoring system that also rewards an individual for capturing the "flow" of the sound event with a coherent story. In our own scoring system, the focus was on identifying key sound components in the correct order and in making simple inferences. Although this is a useful way to index basic identification and understanding of sounds, it is not good for evaluating fluidity of language use. An individual who responds to the "Ping-Pong accident" sound event, for instance, with "Ping-Pong broken glass running" would receive 5 of 7 points for identifying four key components and listing them in the correct order. In contrast, a person with a much more story-like response ("Two people are playing table tennis and then one of them hits the ball too hard. It hits and breaks a window in the attic. They both run out of the room.") would earn the same score in our system. Because there are multiple possible uses for sound event stimuli (such as evaluating auditory agnosia, word-finding difficulties, expressive fluency, or verbal creativity), we echo Marcell et al.'s (2000) suggestions and strongly encourage users to experiment with different scoring systems for specific applications.

\section{ARCHIVED MATERIALS}

The following materials and links may be accessed through the Psychonomic Society's Norms, Stimuli, and Data Archive, www .psychonomic.org/archive.

To access these files or links, search the Archive for this article using the journal (Behavior Research Methods), the first author's name (Marcell), and the publication year (2007).

FILE: Marcell-BRM-2007.zip

DESCRIPTION: The compressed archive file contains two text files and five stimulus files. The two .pdf text files are:

Scoring criteria for sound event identification task.pdf, a $13.5 \mathrm{~K}$ table containing identification task scoring criteria for each of the 22 sound events.

Questions for sound event memory task.pdf, a $42.9 \mathrm{~K}$ table containing memory task multiple-choice questions for each of the 22 sound events.

The five stimulus .zip files contain .wav format versions of the sound event stimuli and are as follows

Experimental stimuli 1-5.zip (5.92 MB)

Experimental stimuli 6-10.zip (7.80 MB)

Experimental stimuli 11-16.zip (5.61 MB)

Experimental stimuli 17-22.zip (6.55 MB)

Practice stimuli 1-2.zip (1.83 MB).

LiNK: www.cofc.edu/ marcellm/sound event studies/sndevent .htm

DESCRIPTION: The three Macromedia Authorware programs used to present the Identification, Rating, and Memory tasks can be run from this link to the author's Web site.

AUTHOR's E-MAIL ADDRESS: marcellm@cofc.edu.

AUTHOR's WeB SITE: www.cofc.edu/ marcellm.

(Manuscript received May 17, 2006; revision accepted for publication June 23, 2006.) 